\title{
Epidemiology and treatment of fungal keratitis in Saint Petersburg, Russia
}

\author{
Bogomolova T.S. ${ }^{1}$, Skryabina E.V. ${ }^{2}$, Chilina G.A. ${ }^{1}$, Vasilyeva N.V. ${ }^{1}$, Klimko N.N. ${ }^{1}$ \\ ${ }^{1}$ Kashkin Research Institute of Medical Mycology NWSMU n.a. I.I. Mechnikov, ${ }^{2}$ City Hospital №2 \\ Saint Petersburg, Russia
}

\section{Objectives}

To study etiology, risk factors and treatment outcomes in patients with fungal keratitis.

\section{Methods}

Diagnosis of fungal keratitis was established by direct fluorescent microscopic examination and culture of corneal scrapings obtained from patients with clinical symptoms of keratitis. Species identification was preliminary based on morphological and biochemical features of fungal cultures and then confirmed by DNA sequencing.

\section{Results}

During 2007-2017 yy. 46 cases of fungal keratitis were observed in Saint Petersburg, Russia. Men to women ratio $1.5: 1$, age range $23-73$. Main risk factors were: contact lenses use $-22(48 \%)$, trauma - 20 (44\%), herpes virus infection - 1, demodicosis - 1, chronic blepharoconjunctivitis 1 , corneal dystrophy - 1 .

At direct microscopy of corneal scrapings the following fungal morphological elements were found: septate hyaline hyphae (63\%), yeast cells and pseudohyphae (22\%), septate brown hyphae $(7 \%)$, non-septate hyaline hyphae (2\%) (Fig. 1). In three cases conidia forming structures of molds were also detected by direct microscopy.

Cultures were positive in $65 \%$ of cases. The etiologic agents of fungal keratitis were: Fusarium spp. (34\%), Aspergillus fumigatus (13\%), Acremonium spp. (10\%), A. flavus (7\%), Penicillium spp. (7\%), Sporothrix schenkii (3\%), Paecilomyces marquandii (3\%), Cladosporium sphaerospermum (3\%), Candida parapsilosis (10\%), C. albicans (7\%), Candida sp. (3\%) (Fig. 2).

Patients received topical (natamycin, amphotericin $B$ ) and systemic (voriconazole, amphotericin B, fluconazole) antifungal therapy in combination with surgical treatment. Clinical improvement was observed in all patients (Fig. 3).
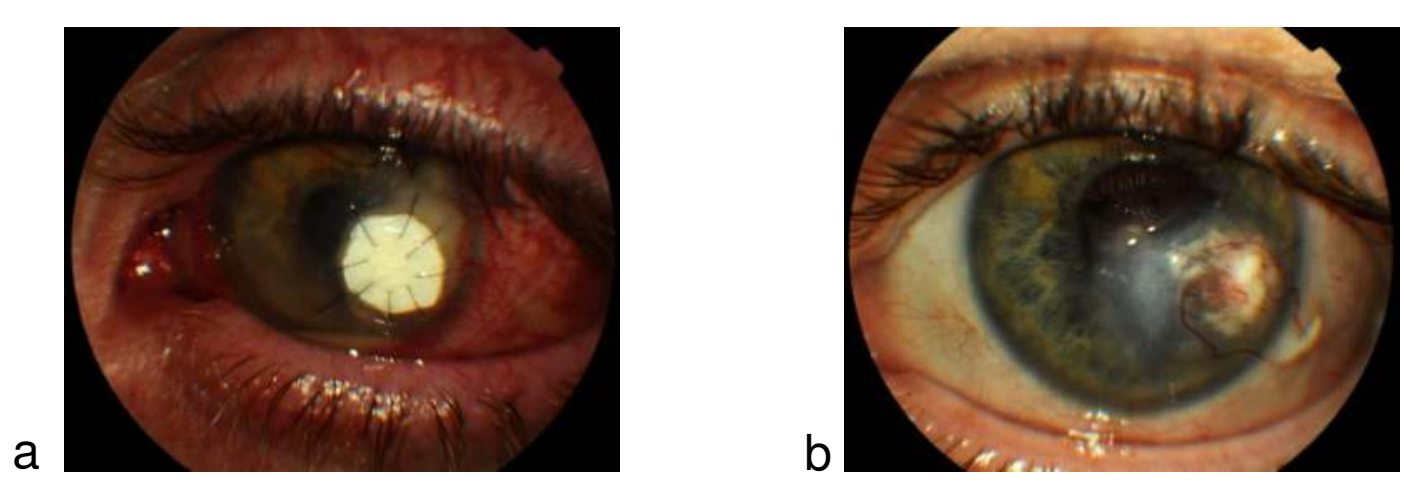

Fig. 3. Clinical picture of keratitis caused by Fusarium sp.: a before treatment; $b$ - after treatment by voriconazole plus surgery.

\section{Conclusion}

The most frequent etiologic agents of fungal keratitis in Saint Petersburg, Russia were Fusarium spp. (34\%). Main risk factors were contact lenses use and trauma of the eye. Combination of antifungal drugs and surgery was an effective treatment of patients with fungal keratitis.

a
Pt. R.

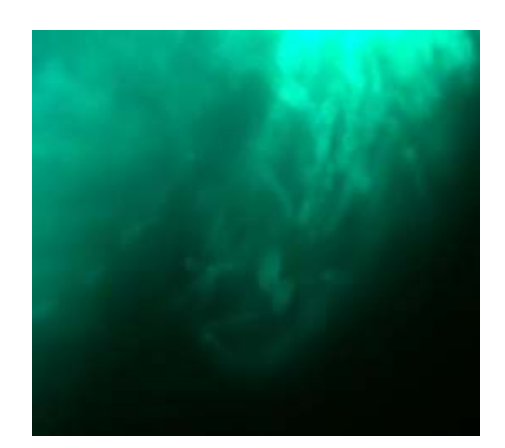

Direct fluorescent microscopy of corneal scrapings. Mag. 400x.

Pt. S.

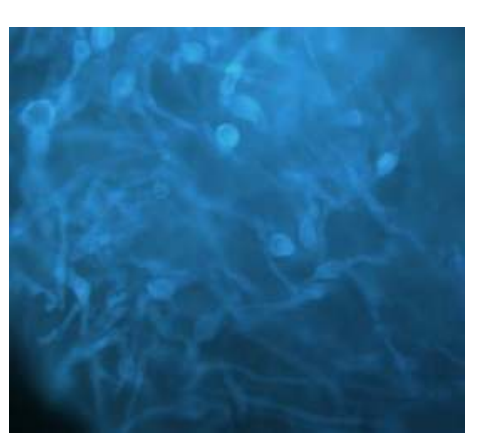

Direct fluorescent microscopy of corneal scrapings. Mag. 400x. Septate hyphae with intercalar swellings.

\section{b}

Pt. I.

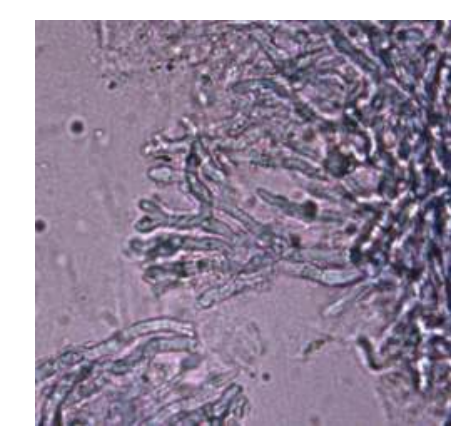

Direct light microscopy o corneal scrapings.

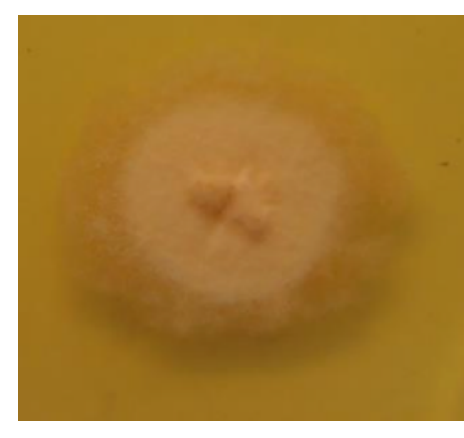

Culture grew Fusarium sp.

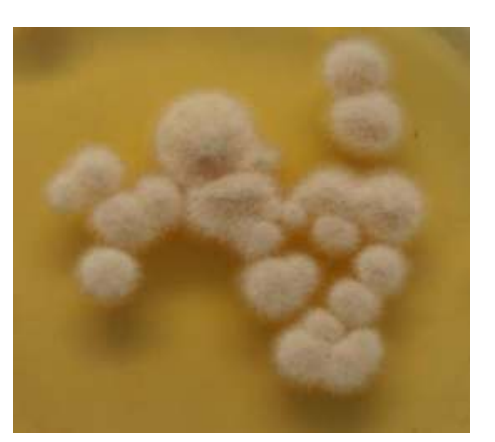

Culture grew Fusarium $s p$.

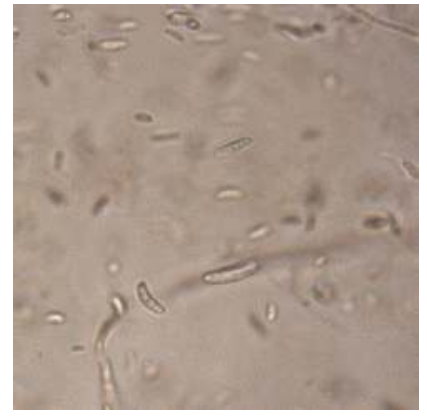

Microscopy of culture. Mag. 400x.
Fig. 1. Morphology of etiologic agents of keratitis at direct microscopy of corneal scrapings and in culture. Mycological findings in patients with culture proven keratitis caused by: a - Fusarium spp., b-Aspergillus fumigatus.

\section{SPECIES DISTRIBUTION OF THE ETIOLOGIC AGENTS OF FUNGAL KERATITIS}

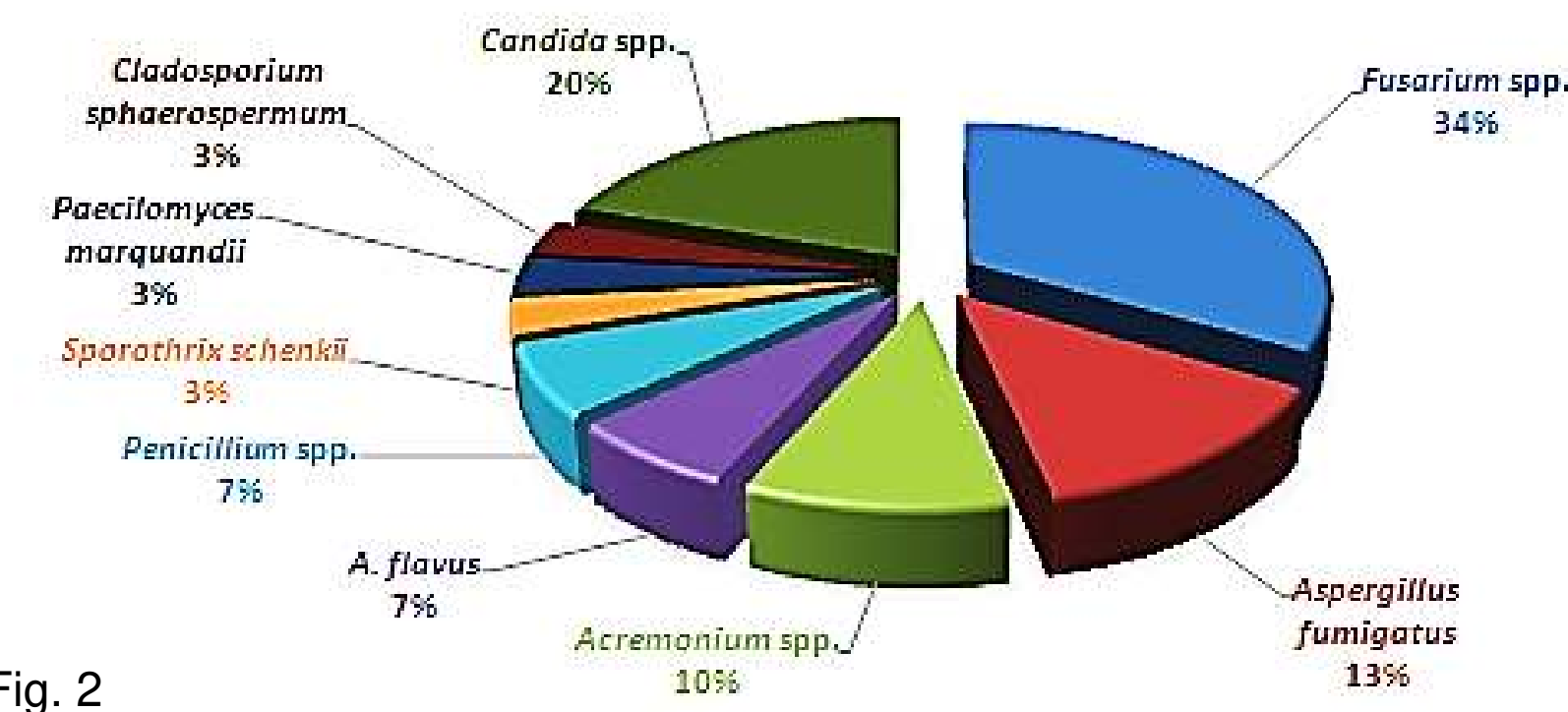

